AC model for Australian men aged 15-29, and 1.9x higher in women. Neither model agreed perfectly with the empirical prevalence estimates; the LW model tended to be closer in younger age-categories and the AC model closer in older agecategories. The AC model was closer to empirical estimates in men than women.

Conclusion Substantial differences were observed between chlamydia prevalence estimates produced by the two models. These findings have important implications for researchers, policymakers and healthcare professionals, as estimation methods must be robust before they are used to inform public health policy, e.g. assessing the impact of chlamydia-control interventions. Health care systems and associated surveillance systems vary by country, and work to understand the reasons for the models' differences is planned, including applying the models to English data, in collaboration with the Universities of Bern, New South Wales, and Otago.

Disclosure No significant relationships.

\section{P460 ASSESSMENT OF TUBAL FACTOR INFERTILITY ATTRIBUTABLE TO CHLAMYDIA WITH PGP3 SEROLOGY}

${ }^{1}$ Gloria Anyalechi*, ${ }^{2}$ Jaeyoung Hong, ${ }^{2}$ Rachel Gorwitz, ${ }^{2} J o h n$ Papp, ${ }^{2}$ Robert Kirkcaldy, ${ }^{3}$ Harold Wiesenfeld, ${ }^{4}$ William Geisler, ${ }^{5}$ Paddy Horner, ${ }^{2}$ Kyle Bernstein. ${ }^{1}$ Centers for Disease Control and Prevention, Division of STD Prevention, Atlanta, USA; ${ }^{2}$ Centers for Disease Control and Prevention, Atlanta, USA; ${ }^{3}$ University of Pittsburgh/Magee-Womens Hospital, Pittsburgh, USA; ${ }^{4}$ University of Alabama at Birmingham, Birmingham, USA; ${ }^{5}$ University of Bristol, Bristol, UK

\subsection{6/sextrans-2019-sti.542}

Background Our recent case-control study explored the Chlamydia trachomatis population attributable fraction (PAF) for tubal factor infertility (TFI) using an elementary body enzymelinked immunosorbent serological assay (EB-ELISA) or a commercially available (Medac) major outer membrane protein ELISA to measure prior chlamydial infection. We examined data from this study using a Pgp3 enhanced ELISA (Pgp3).

Methods In this study of women with TFI by hysterosalpingogram (cases) and non-TFI infertility (controls) in two U.S. infertility clinics, we assessed anti-C. trachomatis seropositivity by Pgp3. We then assessed the association between chlamydia seropositivity and TFI using adjusted odds ratios (aOR) along with 95\% confidence intervals (CI) stratified by race. Finally, the adjusted chlamydia TFI PAF (aPAF) and 95\% CI based on the Pgp3 assay were estimated.

Results All black $(n=107)$ and 618 of 620 non-black women had Pgp3 results. Seropositivity frequency by Pgp3 was 66\% (95\% CI 52-80\%) for black cases, 72\% (60-83\%) for black controls, 26\% (19-33\%) for non-black cases, and 15\% (12$18 \%$ ) for non-black controls. Pgp3 was not associated with TFI among black women (aOR 1.1 [95\% CI $0.4-3.3]$ ). Among non-black women, Pgp3 seropositivity was associated with TFI (aOR 1.8 [95\% CI 1.1-3.0]) adjusting for clinic, age, income, trichomonas, and endometriosis. Using Pgp3 and adjusting for the same variables, chlamydia TFI aPAF was $12 \%(95 \%$ CI 1-22\%) in non-black women.

Conclusion Among non-black women, Pgp3 ELISA seropositivity was associated with TFI. Assays to estimate chlamydia TFI PAF merit further investigation, especially in black women. Chlamydial TFI may be prevented in all women by early identification and treatment of chlamydia.

Disclosure No significant relationships.

\section{P461 BACTERIAL LOAD OF CHLAMYDIA IN THE OROPHARYNX AND SALIVA AMONG GAY AND BISEXUAL MEN WITH UNTREATED OROPHARYNGEAL CHLAMYDIA}

${ }^{1}$ Tiffany Phillips, ${ }^{1}$ Christopher Fairley, ${ }^{1}$ Kate Maddaford, ${ }^{2}$ Jennifer Danielewski, ${ }^{3}$ Jane Hocking, ${ }^{1}$ David Lee, ${ }^{4}$ Deborah Williamson, ${ }^{2}$ Gerald Murray, ${ }^{3}$ Fabian Kong, ${ }^{1}$ Catriona Bradshaw, ${ }^{1}$ Marcus Chen, ${ }^{4}$ Benjamin Howden, ${ }^{1}$ Eric Chow. ${ }^{1}$ Alfred Health, Melbourne Sexual Health Centre, Carlton, Australia; ${ }^{2}$ The Royal Women's Hospital, Centre for Women's Infectious Disease Research, Parkville, Australia; ${ }^{3}$ University of Melbourne, Melbourne School of Population and Global Health, Parkville, Australia; ${ }^{4}$ The University of Melbourne at The Peter Doherty Institute for Infection and Immunity, Microbiological Diagnostic Unit Public Health Laboratory, Parkville, Australia

\subsection{6/sextrans-2019-sti.543}

Background Previous studies have found that saliva can carry infectious gonorrhoea, which has led to the hypothesis that saliva could play an important role in gonorrhoea transmission. However, no study has examined the role of saliva in chlamydia transmission. The aim of this study was to determine whether Chlamydia trachomatis could be detected in saliva and to determine if the infection is specific to an anatomical site; oropharynx or tonsils.

Methods Men who have sex with men (MSM) who tested positive for oropharyngeal chlamydia at Melbourne Sexual Health Centre, who had no antibiotics in the past 4 weeks, and returned for treatment within 14 days between August 2017 and August 2018 were invited to participate. On the day of treatment, throat swabs were taken by clinicians at the tonsillar fossae and another at the posterior oropharynx. A saliva sample was also collected. All samples were tested for Chlamydia by nucleic acid amplification tests. The sample adequacy and bacterial load of Chlamydia trachomatis were assessed by quantitative PCR.

Results Forty-two MSM were included with a median age of 28 (Interquartile range [IQR]:25-33). The majority of men $(76.2 \% ; n=32)$ tested positive at both the tonsils and the oropharynx, followed by $9.5 \%(n=4)$ positive at the oropharynx only, and $4.8 \%(n=2)$ positive at the tonsils only. Chlamydia was detected in saliva in two-thirds of men $(68.0 \% ; n=29)$. The median bacterial load of chlamydia was 446 copies $/ \mathrm{ml}$ (IQR: 204-1390 copies/ml) in saliva, 1230 copies/ml (IQR: 538-18200 copies $/ \mathrm{ml}$ ) from the tonsils and1660 copies $/ \mathrm{ml}$ (IQR: 456-22400 copies/ml) at the oropharynx. The chlamydia loads did not differ between the tonsils and the oropharynx $(\mathrm{p}=0.865)$.

Conclusion Chlamydia can be detected in saliva in most of oropharyngeal chlamydia cases among MSM. Sampling both the tonsils and oropharynx is important for optimal detection of oropharyngeal chlamydia.

Disclosure No significant relationships.

\section{P462 RE-TESTING FOR CHLAMYDIA IN THE NATIONAL CHLAMYDIA SCREENING PROGRAMME IN BRISTOL, ENGLAND: AN ANALYSIS OF SURVEILLANCE DATA}

\footnotetext{
${ }^{1}$ Katherine Davis*, ${ }^{1}$ Joanna Lewis, ${ }^{2}$ Karl Liva-Pye, ${ }^{2}$ Andrew Liebow, ${ }^{3}$ Paddy Horner ${ }^{1}$ Imperial College London, Department of Infectious Disease Epidemiology, London, UK ${ }^{2}$ University Hospitals Bristol NHS Foundation Trust, Unity Sexual Health, Bristol, UK; ${ }^{3}$ University of Bristol, Population Health Sciences, Bristol, UK
}

10.1136/sextrans-2019-sti.544

Background England's National Chlamydia Screening Programme (NCSP) recommends that sexually active people $<25$ 